\title{
Efeito da glicose e sucção não nutritiva na dor de prematuros na punção: ensaio clínico crossover*
}

\author{
Effect of glucose and non-nutritive sucking on puncture pain \\ in premature infants: a crossover clinical trial \\ Efecto de la glucosa y la succión no nutritiva sobre el dolor por \\ pinchazos prematuros: un ensayo clínico cruzado
}

Como citar este artigo:

Silveira ALD, Christoffel MM, Velarde LGC, Rodrigues EC, Magesti BN, Souza RO. Effect of glucose and non-nutritive sucking on puncture pain in premature infants: a crossover clinical trial. Rev Esc Enferm USP. 2021;55:e03732. https://doi.org/10.1590/S1980-220X2020018303732

\section{Ana Luiza Dorneles da Silveira ${ }^{1}$ \\ Marialda Moreira Christoffel ${ }^{2}$ \\ Luis Guillermo Coca Velarde ${ }^{3}$ \\ Elisa da Conceição Rodrigues ${ }^{2}$ \\ Bruna Nunes Magesti ${ }^{4}$ \\ Rebecca de Oliveira Souza ${ }^{5}$}

* Extraído da tese: "O uso da glicose 25\%

e sucção não nutritiva no alívio da dor do pré-termo submetido a punção do calcanhar: um ensaio clínico", Programa de

Pós-Graduação em Enfermagem, Universidade Federal do Rio de Janeiro, 2015.

${ }^{1}$ Universidade Federal Fluminense, Escola de Enfermagem Aurora de Afonso Costa, Departamento de Enfermagem MaternoInfantil e Psiquiátrico, Niterói, RJ, Brasil.

${ }^{2}$ Universidade Federal do Rio de Janeiro, Escola de Enfermagem Anna Nery,

Departamento de Enfermagem Materno-

Infantil, Rio de Janeiro, RJ, Brasil.

${ }^{3}$ Universidade Federal Fluminense,

Departamento de Estatística, Niterói, RJ, Brasil.

${ }^{4}$ Universidade Federal do Rio de Janeiro, Escola de Enfermagem Anna Nery, Programa de Pós-Graduação em Enfermagem, Rio de Janeiro, RJ, Brasil.

${ }^{5}$ Universidade Federal do Rio de Janeiro, Programa de Pós-Graduação em Estatística do Instituto de Matemática, Rio de Janeiro, RJ, Brasil.

\begin{abstract}
Objective: To compare the effect of non-nutritive sucking, $25 \%$ oral glucose and $25 \%$ oral glucose combined with non-nutritive sucking in pain relief in premature infants submitted to heel puncture for blood glucose monitoring. Method: This is a randomized crossover clinical trial with 34 preterm infants who randomly received interventions: non-nutritive sucking, $25 \%$ oral glucose and the two interventions combined for three consecutive days in heel puncture for blood glucose monitoring. Assessment by the Premature Infant Pain Profile for 30 seconds before the intervention, called the baseline period and for 5 minutes after puncture. Results: The combination of interventions made the premature infants return to baseline, with 1 minute and 30 seconds after heel puncture, promoting a $2.2 \%$ percentage reduction in the scale. Conclusion: Comparing the effect of isolated and combined interventions showed that, when offered in combination, preterm infants were able to return to baseline parameters more quickly. Brazilian Clinical Trials Registry: RBR-3gm6w5.
\end{abstract}

\section{DESCRIPTORS}

Pain; Infant, Premature; Punctures; Sucking Behavior; Glucose; Neonatal Nursing. 


\section{INTRODUÇÃO}

A dor de recém-nascidos prematuros (RNPT) no ambiente da Unidade de Terapia Intensiva Neonatal (UTIN) é foco de preocupação e controle em decorrência das flutuações hemodinâmicas que provoca, levando a sequelas em curto e longo prazos ${ }^{(1)}$, tais como instabilidade fisiológica, alteração no desenvolvimento cerebral, resposta sistêmica ao estresse e padrões de anormalidade no neurodesenvolvimento ${ }^{(2)}$. Entre as causas frequentes de dor, pode se destacar a regularidade da punção do calcanhar, devido à necessidade de monitorização da glicemia $^{(1,3)}$. No estudo que observou 562 recém-nascidos por 14 dias enquanto internados na UTIN, foram identificadas 8.995 punções do calcanhar, dentre as quais 7.922 realizadas para monitorização da glicemia. Assim, cada recém-nascido foi submetido a uma média de 2,1 punções por dia, com uma variação de 0 a $20^{(1)}$.

A avaliação da dor deve ser realizada com instrumentos de medida validados de forma contínua, para que possa nortear os profissionais a promover o seu alívio de forma efetiva. Dentre as escalas recomendadas, a Premature Infant Pain Profile (PIPP) engloba, além de medidas fisiológicas e respostas comportamentais a dor, os fatores contextuais idade gestacional e estado comportamental ${ }^{(2)}$. Esses fatores contextuais consideram os aspectos do desenvolvimento neurológico diretamente relacionado à idade gestacional $\mathrm{e}$ à capacidade de responder à dor, assim como o estado de sono e atividade, que são influenciados pelo estímulo doloroso, ampliando a reflexão sobre a definição de dor para o recém-nascido ${ }^{(4)}$.

O alívio da dor para os procedimentos deve ser planejado prioritariamente e realizado com intervenções não farmacológicas inicialmente ${ }^{(2-3)}$, dentre as quais as soluções adocicadas e a sucção não nutritiva (SNN) são estratégias bem estabelecidas e recomendadas ${ }^{(1-3)}$. A glicose na concentração entre 20 e 30\% é considerada efetiva, segura e com limitados efeitos colaterais no alívio da dor, entretanto não há consenso quanto à dose ideal ${ }^{(2-3)}$. No Brasil, a glicose $25 \%$ tem sido mais utilizada na prática clínica, por ser facilmente encontrada na forma de ampola manufaturada e comercializada para unidades hospitalares.

A SNN é considerada uma intervenção que promove a auto-regulação e estabilidade fisiológica nos $\mathrm{RNPT}^{(5)}$. Quando combinada com a solução adocicada, mostra um efeito aditivo que produz impacto direto na redução de respostas comportamentais e fisiológicas de RNPT, assim como na pontuação referente a dor avaliada por escalas ${ }^{(6)}$. Por outro lado, não difere do uso da glicose isolada na redução dos riscos de bradicardia, taquicardia e incidência de dessaturação ${ }^{(6)}$.

Comumente, os estudos que abordam o uso de intervenções não farmacológicas no alívio da dor se destinam a um determinado momento e único procedimento. Assim, neste estudo, foi estabelecida como hipótese que a combinação da glicose $25 \%$ com a SNN seja superior ao uso dessas, de forma isolada, no alívio da dor de RNPT submetidos à punção do calcanhar para monitorização da glicemia, por três dias consecutivos.
O objetivo foi comparar o efeito da SNN, glicose oral $25 \%$ e glicose oral $25 \%$ combinada com a SNN no alívio da dor de RNPT submetidos à punção do calcanhar, para monitorização da glicemia.

\section{MÉTODO}

\section{TIPO DE ESTUdo}

Trata-se de um ensaio clínico randomizado crossover, desenvolvido na UTIN da Maternidade Escola da Universidade Federal do Rio de Janeiro, no período de março de 2014 a maio de 2015.

\section{PopulaÇão}

A população foi constituída de 94 RNPT admitidos na UTIN com idade gestacional ao nascer entre 29 e 36 semanas completas e prescrição médica de monitorização da glicemia capilar. Após aplicados os critérios de seleção, foi constituída uma amostra de 34 RNPT.

\section{Critérios de SELEÇão}

Foram incluídos RNPT com Apgar $\geq 7$ no quinto minuto de vida, idade pós natal $\geq 6$ horas de vida, estabilidade clínica com frequência cardíaca e saturação de oxigênio dentro dos parâmetros de normalidade e dieta enteral iniciada. Foram excluídos aqueles com diagnóstico de hemorragia intraventricular grau III ou IV, leucomalácia, alterações cromossômicas, malformações do sistema nervoso central, cardiopatias congênitas, enterocolite necrotizante, hiperglicemia, assistência ventilatória, uso de drogas que interferissem na resposta de nocicepção da dor, mães usuárias de drogas ilícitas, traumas decorrentes do parto e precaução de contato. Foi adotado como critério de descontinuidade e perda a suspensão da monitorização da glicemia por um período menor do que três dias.

\section{INTERVENÇÃO E COLETA DE DADOS}

Cada participante recebeu as três intervenções propostas, 1- SNN, 2- glicose $25 \%$ e 3- glicose $25 \%+$ SNN, uma para cada dia em que a punção do calcanhar foi realizada para monitorização da glicemia. Toda coleta foi realizada no período da tarde após verificação da prescrição médica do dia.

A SNN foi oferecida com dedo mínimo enluvado (luva de vinil sem látex) dois minutos antes e durante a punção, devendo ser mantida frequência $\geq 32$ sucções por minuto. A glicose $25 \%$ foi oferecida no volume de $1 \mathrm{ml}$ diretamente na parte anterior da língua com uma seringa de $1 \mathrm{ml}$ sem agulha, dois minutos antes da punção. A glicose 25\% + SNN foi oferecida dois minutos antes da punção, sendo primeiro oferecida a glicose e depois a SNN, conforme os termos anteriormente descritos.

Para traçar o perfil dos RNPT e da internação, as seguintes variáveis foram investigadas por meio dos prontuários: sexo, peso ao nascer, Apgar no quinto minuto de vida, tipo de parto, idade gestacional ao nascer, assistência ao nascimento, diagnóstico de internação, tempo de ingresso no estudo e procedimentos realizados no período de 24 horas antes da 
punção do calcanhar em cada dia de coleta. Foram listados vinte e cinco procedimentos considerados dolorosos e indolor (estressante), o número de vezes que foram repetidos e a medida de alívio da dor utilizada. Todos os procedimentos listados tiveram como base o instrumento do estudo denominado Epidemiology of Painful Procedures in Neonates (EPIPPAIN), devidamente autorizado pelo autor ${ }^{(7)}$.

A segunda parte do instrumento foi obtida no momento da coleta em cada dia, com identificação da data da avaliação, idade gestacional corrigida, peso atual, medida não farmacológica utilizada, eventos adversos ocorridos, conduta e valor da glicemia. Foi avaliada a dor por meio do PIPP, considerada desfecho primário.

O PIPP, traduzido e adaptado para a língua portuguesa adotada no Brasil e amplamente utilizado em pesquisas clínicas $^{(8)}$, divide-se em dois momentos: observação do recém-nascido por 15 segundos em que se considera como indicador a idade gestacional atual e o estado comportamental. Quanto menor a idade gestacional, maior a pontuação na escala. O estado comportamental a se observar é: se ativo ou quieto, acordado ou sono, olhos fechados ou abertos e movimentos faciais presentes ou ausentes. Este primeiro momento deve ser realizado previamente ao procedimento ser realizado, a fim de registrar o estado comportamental do recém-nascido antes do estímulo doloroso.

O segundo momento se refere à observação do recém-nascido por 30 segundos, considerando os indicadores fisiológicos frequência cardíaca e saturação de oxigênio e três indicadores comportamentais: sobrancelha saliente, olhos espremidos e sulco nasolabial. A pontuação total da escala é de 21 pontos, em que valores menores ou igual a seis indicam dor mínima ou ausente; entre 7 e 11, dor presente; maior ou igual a 12 , dor moderada a intensa. Neste segundo momento, são verificados o tempo de permanência de cada um dos indicadores comportamentais e as flutuações dos indicadores fisiológicos após o estímulo doloroso. Quanto maior o tempo de permanência do indicador comportamental e mais intensa a alteração do indicador fisiológico, maior a pontuação. Utilizou-se como parâmetro a pontuação do instrumento no período basal.

Para obtenção da pontuação do PIPP, cada RNPT foi observado por 30 segundos antes da aplicação da intervenção considerado como período basal e por cinco minutos a contar do momento da punção do calcanhar para coleta do sangue. A pontuação foi obtida por cada bloco de 30 segundos. $O$ primeiro minuto foi denominado recuperação imediata (RI), e o tempo após, recuperação tardia (RT). Os tempos correspondentes aos blocos estabelecidos foram RI30, RI60, RT90, RT120, RT150, RT180, RT210, RT240, RT270 e RT300.

Foram utilizadas duas câmeras filmadoras digitais, uma fixa e direcionada para o monitor de oximetria de pulso e outra diretamente para a face do participante, manuseada pela pesquisadora principal no momento da coleta. Um frequencímetro Polar RS800X foi conectado a dois eletrodos cardíacos na região torácica do RNPT para verificação da frequência cardíaca; posteriormente, foi transferido para uma planilha digital própria do equipamento para verificação da flutuação dos resultados. Foi utilizado o software Magix
Movie Edit Pro 15 Plus para efetuar a codificação segundo a segundo dos movimentos faciais (sobrancelhas salientes, olhos espremidos e sulco nasolabial), registrando em uma planilha o tempo que cada um dos movimentos foi mantido dentro dos intervalos estabelecidos do período basal e cinco minutos após a punção do calcanhar. Foi verificada também a saturação de oxigênio e registrada em folha própria.

Durante a coleta, alguns participantes apresentaram condições com potencial de confundir a resposta a dor, tais como o uso da cafeína e a frequência de sucções insuficientes. Para controlar tais condições, foi realizada análise com a "intenção de tratar" por meio de um modelo hierárquico de regressão sob um enfoque bayesiano. Todas as variáveis identificadas como potencialmente influentes na resposta do RNPT em cada dia de avaliação foram controladas, tais como peso, frequência das sucções, uso da cafeína, intervenção não farmacológica utilizada, idade gestacional corrigida, quantitativo de procedimentos dolorosos e quantitativo de procedimentos estressantes.

\section{DEFINICCÃO DA AMOSTRA}

Para efetuar o cálculo do tamanho amostral, foi considerado que todas as intervenções estariam sendo efetivas, conforme o apontado na literatura. Assim, para que a hipótese alternativa fosse respondida, foi definida uma diferença na pontuação da escala que deveria ser obtida entre o uso das intervenções isoladas e combinadas. A magnitude de efeito das intervenções isoladas e combinadas foi considerada com uma margem de redução de três pontos no total do PIPP, para que a combinação da glicose $25 \%$ com a SNN fosse mais efetiva do que as intervenções isoladas, de acordo com estudos anteriores ${ }^{(9-10)}$. Foram adotados como parâmetros um nível de significância de $5 \%$ e poder do teste de $80 \%$, com o total de 34 participantes. Considerando a probabilidade de perdas durante o experimento, efetuou-se um acréscimo de $20 \%$ ao tamanho da amostra inicial, totalizando 40 RNPT.

A randomização foi realizada por meio de técnica simples através do site www.randomization.com. O plano individual gerado poderia obedecer às seguintes sequências: 1,2 e 3 ou 1, 3 e 2 ou 2, 3 e 1 ou 2, 1 e 3 ou 3, 2 e 1 ou 3, 1 e 2 . Cada plano individual foi inserido em um envelope opaco, lacrado e numerado consecutivamente, sendo utilizado de acordo com a entrada do recém-nascido no estudo. Para cada participante que ingressou no estudo, foi atribuído o número correspondente ao do envelope lacrado e oferecida a intervenção randomizada para o dia correspondente da coleta, totalizando três coletas.

O plano de randomização foi realizado por uma auxiliar de pesquisa que não esteve envolvida com a coleta. Uma segunda auxiliar de pesquisa foi a responsável por oferecer as intervenções não farmacológicas previamente ao procedimento de acordo com a sequência no envelope correspondente ao participante. Todas as punções do calcanhar para coleta do sangue foram realizadas por técnicas de enfermagem assistenciais da própria unidade. O cegamento não foi possível de ser realizado em decorrência do uso da SNN antes e durante o procedimento de punção do calcanhar. (Figura 1) 

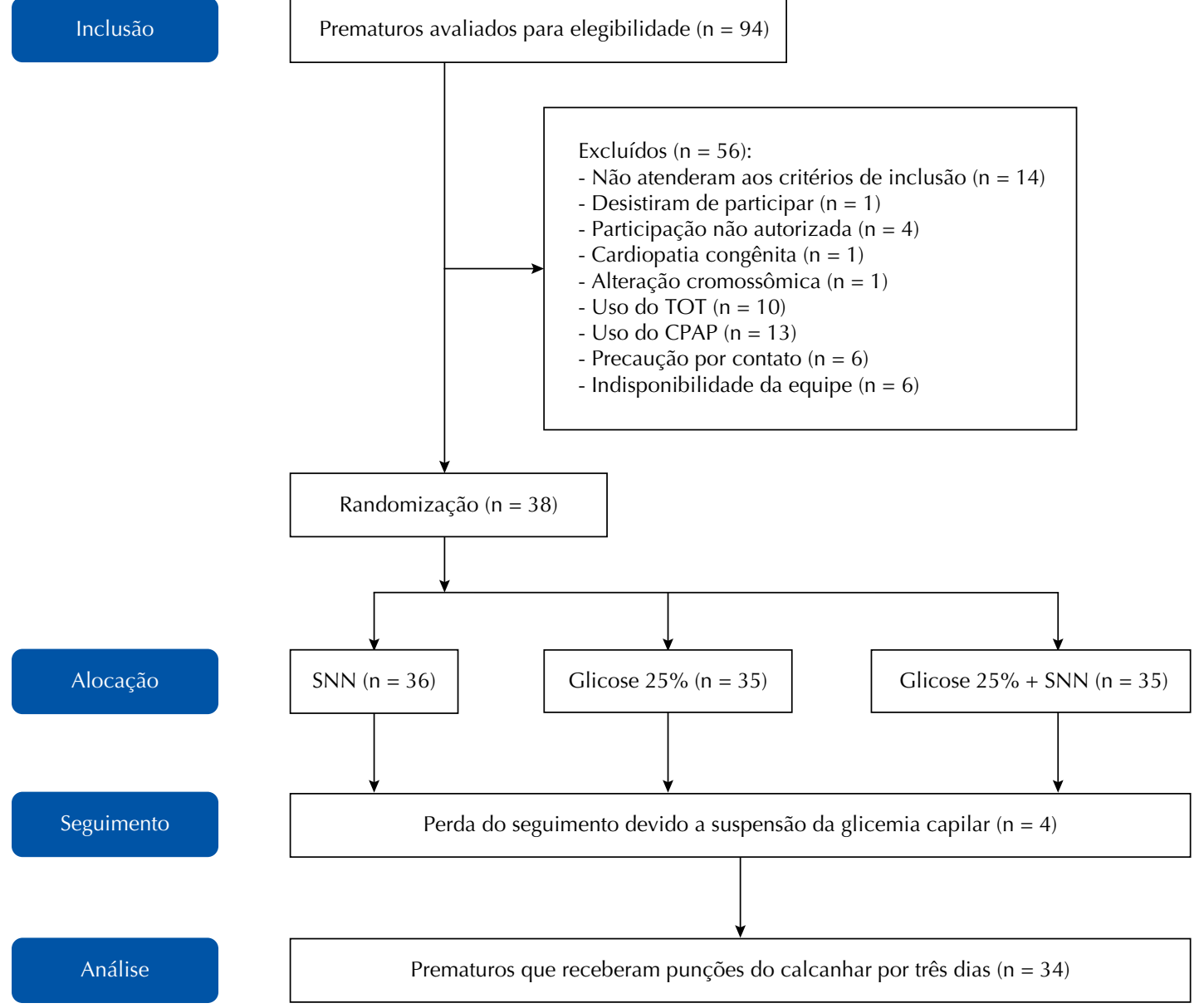

Fonte: Adaptado segundo Consort (http://www.consort-statement.org/consort-statement/flow-diagram).

Figura 1 - Fluxograma de rastreamento da amostra.

\section{ANÁlISE E TRATAMENTO DOS DADOS}

Todos os dados coletados foram submetidos à dupla digitação por pesquisadores independentes; posteriormente, foi testada sua consistência, com a elaboração do banco em planilha no Microsoft Office Excel, versão 2007. Para obtenção da confiabilidade interobservadores, foram codificados os movimentos faciais de $10 \%$ da amostra por duas enfermeiras previamente treinadas e cegadas quanto aos objetivos do estudo, sendo obtido KAPPA de 90\% para sobrancelhas salientes e $100 \%$ para olhos espremidos e sulco nasolabial.

A princípio, foi aplicada estatística descritiva com medidas de tendência central e de dispersão para caracterização da amostra. Para a comparação inicial da pontuação total, obtida pelo PIPP com o uso das intervenções glicose $25 \%$, SNN ou glicose $25 \%+$ SNN, foram realizados os testes t de Student e não paramétrico de Wilcoxon pareado, a fim de obter o valor de p. Testes de comparação com valor de $\mathrm{p}<0,05$ indicaram que não houve retorno ao período basal, considerando que, de acordo com a literatura, todas as intervenções não farmacológicas utilizadas neste estudo seriam efetivas.

A partir da punção do calcanhar, foi obtida uma pontuação pelo PIPP em cada bloco de 30 segundos, sendo consideradas as variações de um valor médio atribuído a cada dia de avaliação para cada RNPT, do efeito do tempo do momento do procedimento doloroso até a conclusão, além da condição do RNPT ser pequeno para idade gestacional ou não.

Para efetuar os cálculos referentes à análise quantitativa, foi utilizado o software OpenBUGS, versão 3.2.3 Rev, 1012, disponível em http://www.openbugs.net.

A seguir, é apresentado o modelo hierárquico utilizado. Neste, Y representa a pontuação PIPP do bebê (i) submetido à intervenção $(\mathrm{j})$ no instante (tempo) (k). Os coeficientes "alpha" representam o efeito numérico da correspondente variável. (Figura 2)

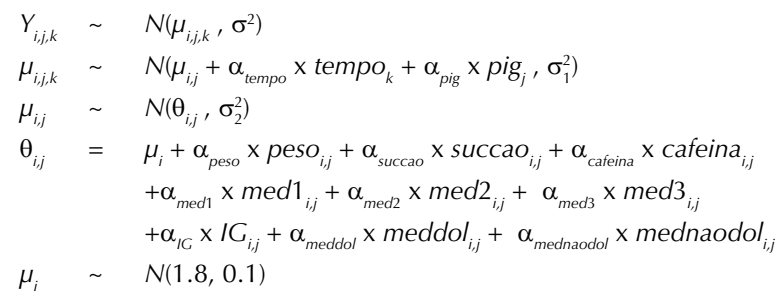

Figura 2 - Equações referentes ao modelo hierárquico bayesiano. 


\section{Aspectos Éticos}

Os responsáveis autorizaram a participação dos RNPT por meio da assinatura do Termo de Consentimento Livre e Esclarecido (TCLE). O projeto foi aprovado pelos Comitês de Ética das instituições proponente e co-participante nos meses de outubro e dezembro do ano de 2013, sob os pareceres 439.157 e 492.643 respectivamente. Obteve-se o registro RBR-3gm6w5 pelo Registro Brasileiro de Ensaios Clínicos (ReBEC).

\section{RESULTADOS}

Os dados apresentados na Tabela 1 mostram o perfil dos recém-nascidos e da internação enquanto na UTIN. Não houve predominância de sexo; os RNPT nasceram, em média, com 33 semanas e 5 dias de idade gestacional e baixo peso por parto cesáreo; a maioria não necessitou de suporte de oxigênio na sala de parto. $\mathrm{O}$ diagnóstico de internação predominante foi a prematuridade, associada ao desconforto respiratório, e o tempo de ingresso no estudo foi de 10 horas de vida a seis dias.

No primeiro dia de coleta, os RNPT estavam, em média, com 34 semanas (sem) e 1 dia de vida; no segundo dia, 34 semanas e 1 dia; no terceiro dia, 34 semanas e 3 dias, mantendo baixo peso em todos os dias. Foram verificados dez episódios de eventos adversos descritos na Tabela 1 , seis episódios na administração da glicose $25 \%$, três após administração da glicose $25 \%$ combinada com a SNN e um episódio enquanto se oferecia a SNN. Em todos os episódios, houve recuperação espontânea após uma pausa no oferecimento das intervenções.

Com relação aos procedimentos dolorosos realizados nas 24 horas que antecederam as coletas da glicemia, foi identificada uma média de 13,6 procedimentos por RNPT no primeiro dia de coleta. Nos $2^{\circ}$ e $3^{\circ}$ dias de coleta, foi uma média de 9 procedimentos dolorosos por RNPT por dia. Dentre os procedimentos dolorosos identificados, a punção do calcanhar foi a mais frequente, sendo realizada 150 vezes no $1^{\circ}$ dia de coleta, com média de 4,4 punções por RNPT, 155 vezes no $2^{\circ}$ dia de coleta, com média de 4,0 por RNPT e 127 vezes no $3^{\circ}$ dia de coleta, com média de 3,7 por RNPT. Dos procedimentos estressantes, a troca de fraldas foi a mais frequente, sendo realizada 259 vezes no primeiro dia, com média de 7,6 vezes por RNPT, 287 vezes no segundo dia, com média de 8,4 vezes/RNPT e 279 no terceiro dia, com média de 8,2 vezes por RNPT.

Foi identificado, isoladamente no prontuário de quatro RNPT, o uso de intervenções para o alívio da dor na instalação do cateter central de inserção periférica (CCIP) e punção venosa para coleta de sangue. No procedimento de inserção do CCIP, dois RNPT receberam fentanyl nas 24 horas que antecederam o $2^{\circ}$ dia de coleta, além de um destes receber também o enrolamento. No procedimento de punção venosa para coleta de sangue, um RNPT recebeu a glicose $25 \%+$ SNN nas 24 horas antecedentes do $1^{\circ}$ dia de coleta, e um RNPT, no $3^{\circ}$ dia de coleta.
Tabela 1 - Caracterização dos recém-nascidos prematuros quanto ao nascimento e período de internação enquanto nos dias de coleta de dados - Rio de Janeiro, RJ, Brasil, 2015.

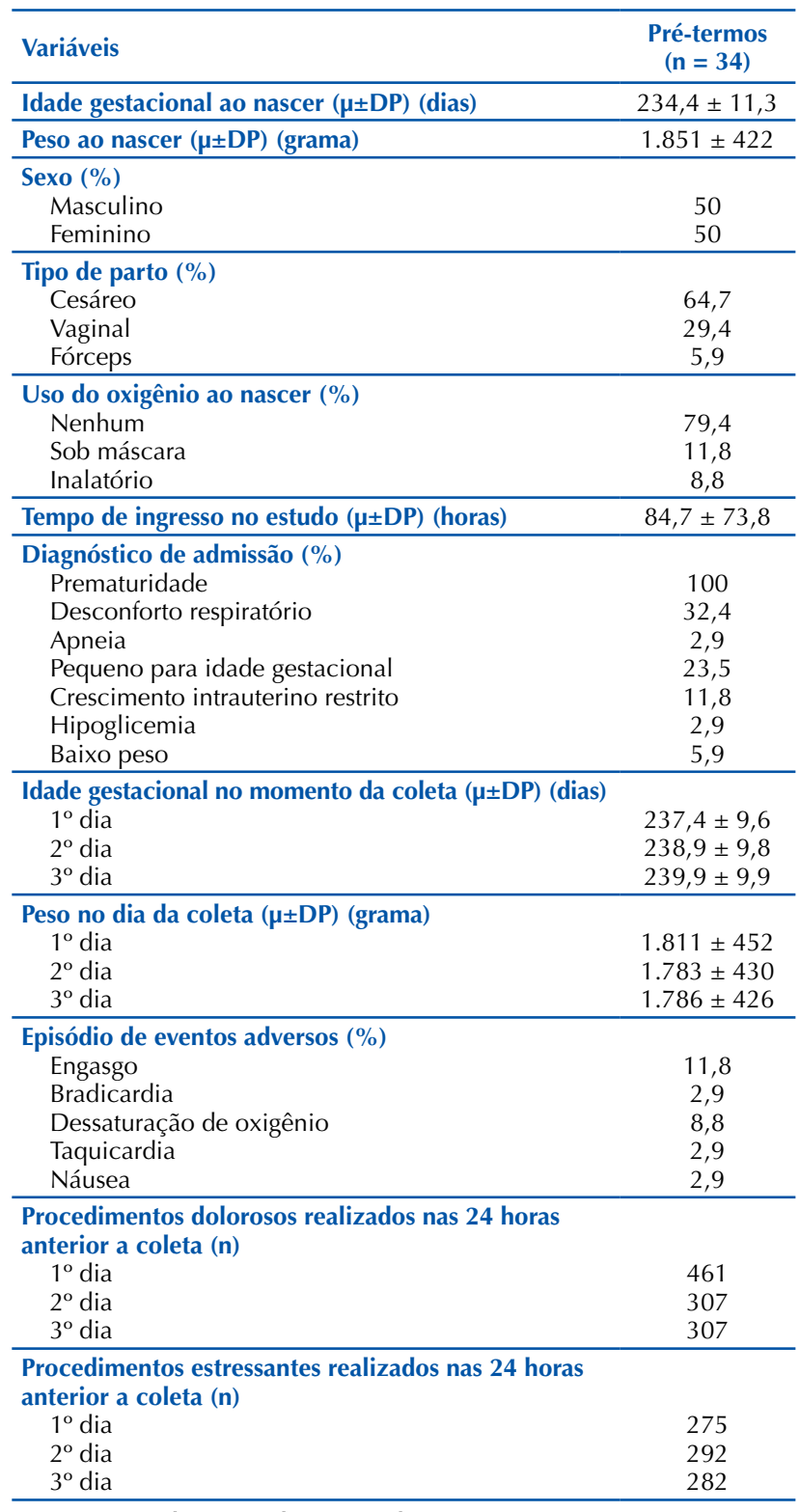

Nota: $\mu$ - média; DP - desvio padrão.

A Tabela 2 analisa a pontuação PIPP para cada intervenção oferecida em cada tempo estabelecido. Além disso, são apresentados os resultados dos testes de hipótese que comparam a média ou mediana da pontuação PIPP de cada tempo com o tempo basal. No caso da SNN e glicose $25 \%$, há indícios de que as médias de todos os tempos têm diferença significativa da média do tempo basal (valor de $\mathrm{p}<0,05$ ), ou seja, as intervenções isoladas não foram capazes de fazer com que os RNPT voltassem aos mesmos parâmetros identificados no período basal, antes do procedimento doloroso ser realizado, quando não apresentavam dor. Para as duas intervenções combinadas, não há evidências de diferenças entre as médias a partir do tempo RT 90 com a do período basal (valor de $\mathrm{p}>0,05$ ). Sendo assim, mostram-se mais efetivas porque fazem com que os RNPT retornem ao parâmetro basal após um minuto e trinta segundos da punção. 
Tabela 2 - Análise do efeito das intervenções não farmacológicas sucção não nutritiva, glicose $25 \%$ e sucção não nutritiva combinada com glicose $25 \%$ no procedimento de punção do calcanhar segundo a média da pontuação do PIPP para cada tempo - Rio de Janeiro, RJ, Brasil, 2015.

\begin{tabular}{|c|c|c|c|c|c|c|c|c|c|}
\hline \multirow{3}{*}{ Tempos } & \multicolumn{9}{|c|}{ Intervenção } \\
\hline & \multicolumn{3}{|c|}{ SNN } & \multicolumn{3}{|c|}{ Glicose $25 \%$} & \multicolumn{3}{|c|}{ SNN + Glicose $25 \%$} \\
\hline & $\mu$ & DP & Valor de $p^{a}$ & $\mu$ & DP & Valor de $p^{b}$ & $\mu$ & DP & Valor de $\mathrm{p}^{\mathrm{a}}$ \\
\hline PB (Base) & 3,3 & 1,5 & - & 3,3 & 1,1 & - & 4,0 & 2,5 & - \\
\hline RI 30 & 8,0 & 3,6 & $<0,0001$ & 8,2 & 4,3 & $<0,0001$ & 6,6 & 3,7 & 0,0015 \\
\hline RI 60 & 6,3 & 3,3 & $<0,0001$ & 6,2 & 3,1 & $<0,0001$ & 5,1 & 2,3 & 0,0079 \\
\hline RT 90 & 5,4 & 2,0 & $<0,0001$ & 5,3 & 2,1 & $<0,0001$ & 4,5 & 2,3 & 0,9765 \\
\hline RT 120 & 5,0 & 1,8 & 0,0001 & 5,1 & 2,0 & $<0,0001$ & 4,2 & 2,0 & 0,1464 \\
\hline RT 150 & 5,0 & 2,2 & 0,0003 & 4,9 & 2,0 & $<0,0001$ & 4,0 & 1,9 & 0,2020 \\
\hline RT 180 & 4,8 & 2,2 & 0,0003 & 5,1 & 2,2 & $<0,0001$ & 4,1 & 1,9 & 0,2127 \\
\hline RT 210 & 4,7 & 1,8 & 0,0009 & 4,8 & 1,8 & $<0,0001$ & 4,4 & 2,2 & 0,0684 \\
\hline RT 240 & 5,1 & 1,8 & 0,0001 & 4,9 & 2,3 & $<0,0001$ & 4,5 & 2,0 & 0,0459 \\
\hline RT 270 & 4,8 & 2,0 & 0,0001 & 4,8 & 2,2 & $<0,0001$ & 4,0 & 1,7 & 0,3100 \\
\hline RT 300 & 5,5 & 3,0 & $<0,0001$ & 4,6 & 2,1 & 0,0003 & 4,2 & 2,4 & 0,1079 \\
\hline
\end{tabular}

Nota: ${ }^{a}$ Teste não paramétrico Wilcoxon pareado; ${ }^{\mathrm{b}}$ Teste t de Student; $\mu$ - média; DP - desvio padrão.

Com relação às variáveis confundidoras, que foram controladas pelo modelo de regressão sob enfoque bayesiano, o que se pôde observar foi uma tendência de redução da dor por meio da pontuação PIPP, quando sob influência do uso da cafeína oral (redução de $2 \%$ no PIPP), do número de procedimentos estressantes (redução de 6\% no PIPP), do peso (redução de $1 \%$ no PIPP quanto maior o peso), do tempo de observação do procedimento (redução de $1 \%$ no PIPP quanto maior for o tempo decorrido após o procedimento doloroso), de ser pequeno para a idade gestacional (redução de 16\% no PIPP) e do uso combinado da glicose oral $25 \%$ com a SNN (redução de $2,2 \%$ no PIPP). Houve tendência de aumento da dor com o uso isolado da glicose $25 \%$ (aumento de $15,4 \%$ no PIPP), uso isolado da SNN (aumento de 12,4\% no PIPP), número de procedimentos dolorosos (aumento de 0,5\% no PIPP) e sucção insuficiente (aumento de $15 \%$ no PIPP).

Foi estimado o valor médio esperado para o PIPP no primeiro dia de coleta do RNPT 27, que foi de 6,1. O cálculo estimado foi referente ao último bloco de avaliação (RT300), em que foram perdidos os dados devido à conclusão do estudo com quatro minutos e trinta segundos ao invés de cinco minutos. O intervalo de credibilidade aponta para uma grande margem de incerteza.

\section{DISCUSSÃO}

No presente estudo, o que se pôde observar é que o uso da glicose oral $25 \%$ combinada com a SNN foi a intervenção que fez com que os RNPT retornassem à pontuação do PIPP ao mesmo valor que o obtido no período basal, um minuto e trinta segundos após a punção do calcanhar. Ao passo que, quando foram utilizadas a $\mathrm{SNN}$ e a glicose oral $25 \%$ isoladas, não houve equiparação da pontuação pelo PIPP com o do momento basal até a conclusão da observação, que foi de cinco minutos. Isso significa que a combinação da glicose oral $25 \%$ com a SNN utilizada para o alívio da dor no procedimento de punção do calcanhar favorece o retorno ao estado basal mais rápido.
Entretanto, a diferença da pontuação PIPP esperada para que a superioridade da intervenção fosse confirmada não foi verificada estatisticamente. Além disso, o uso da glicose $25 \%$ e SNN de forma isolada mostrou aumento de 15,4\% e 12,4\% na pontuação total do PIPP, respectivamente, contrariando o disposto na literatura.

Algumas razões para que os resultados do modelo estatístico não tenham tido o impacto esperado podem estar relacionados à falta de um grupo controle, em que as condições de realização do procedimento a ser adotada fosse a da rotina da unidade, permitindo comparações além do parâmetro basal. Outro fator que limitou o estudo foi o quantitativo de RNPT que não sugou efetivamente e que optamos por não excluir do estudo. Um número de participantes em condições ideais como a prevista pelo cálculo amostral poderia mostrar melhores resultados na análise dos dados, contudo optamos por retratar a realidade individual dos RNPT nos três dias que foram avaliados e oferecer, ao longo do texto, opções para aliviar a dor, tendo como parâmetro o período basal, utilizado como referência para avaliações posteriores.

Estudos que indicam e avaliam a dor por meio de escalas ${ }^{(1-10)}$ consideram o período basal como um parâmetro, para que as alterações multidimensionais sejam verificadas. A observação sistemática auxilia na análise do tempo que foi dispensado para que os mesmos parâmetros fossem alcançados, com o uso de intervenções para o alívio da dor frente ao estímulo aplicado. Assim, para que uma intervenção seja considerada efetiva, o tempo contabilizado do término do estímulo à auto regulação deve ser o menor possível, refletido na pontuação da escala.

Resultados semelhantes foram encontrados no ensaio clínico ${ }^{(11)}$ realizado com 86 RNPT, com média de 31,7 semanas de idade gestacional. A eficácia e segurança da $\mathrm{SNN}$ e a sacarose foram avaliadas em três punções do calcanhar não consecutivas, para monitorização da glicemia, como rotina do tratamento. Os RNPT que utilizaram as intervenções combinadas não sentiram dor, e os que utilizaram as substâncias isoladas sentiram dor leve ${ }^{(11)}$. Apesar dos resultados, todas as opções 
descritas para o alívio da dor são seguras ${ }^{(2-6,11)}$, recomendadas ${ }^{(1-6)}$ e podem ser oferecidas mediante o planejamento prévio das ações pelo profissional e estabelecimento de protocolos institucionais, cabendo a avaliação da situação de cada RNPT ${ }^{(2)}$.

Procedimentos terapêuticos e clínicos realizados durante a internação dos RNPT precisam ser previamente avaliados quanto à real necessidade, visto que estudos recomendam ${ }^{(2-3)}$ que procedimentos dolorosos sejam evitados, que o uso da monitorização não invasiva seja preconizado, assim como antever a necessidade de futuros estudos. Tratando-se da necessidade de realização do procedimento doloroso, deve-se planejar o uso de intervenção não farmacológica.

Em estudo ${ }^{(12)}$ que revisou os tratamentos com intervenções não farmacológicas frequentemente utilizadas em procedimentos dolorosos que envolvessem o rompimento da pele, mostrou-se que o efeito aditivo das soluções adocicadas com a SNN é eficaz para dor leve e moderada. Entretanto, quanto mais procedimentos dolorosos foram realizados, analgésicos mais fortes foram utilizados, fortalecendo a necessidade do controle do número de procedimentos dolorosos para que forneça parâmetros para o profissional. Uma alternativa para o controle do número de procedimentos é o registro diário e individualizado dos procedimentos dolorosos e estressantes realizados, assim como as tentativas efetuadas até a conclusão.

Embora as recomendações sejam claras com relação à redução do quantitativo de procedimentos e ao uso de medidas para o alívio da dor dos RNPT, as ações ainda estão aquém. Em estudo transversal retrospectivo ${ }^{(13)}$ de prontuários, foi verificado que, nos primeiros sete dias de internação na UTIN, os 150 RNPT foram submetidos a 4.765 procedimentos invasivos, sendo uma média de 6,6 por recém-nascido por dia. $\mathrm{O}$ procedimento mais frequente foi a punção do calcanhar, efetuada 1.702 vezes, sendo que não foram encontrados registros de uso de medidas de alívio da dor não farmacológicas prévia, assim como de farmacológicos pela maioria. Quando verificada a aplicação da escala de dor, foram identificados 3.884 registros, em que $96,8 \%$ correspondiam à ausência de dor, e $17,1 \%$, presença de dor, contudo não foram verificadas intervenções para o alívio da dor na maioria.
Estudos $^{(14)}$ que utilizaram métodos não invasivos de imagem da atividade cerebral com intuito de avaliar a eficácia de intervenções não farmacológicas e farmacológicas no alívio da dor mostraram que são ferramentas úteis capazes de verificar que a atividade cerebral pode ser observada, inclusive, na ausência de indicadores de dor, sendo influenciada por fatores contextuais, como idade, sexo, dor prévia, nível de estresse e doença. Neste estudo, fatores como a troca de fraldas, o peso, o uso da cafeína e ser pequeno para idade gestacional refletiram em uma redução na pontuação do PIPP, que poderiam ser melhor explorados em estudos com abordagem multimodal.

Assim como os fatores contextuais, outros ${ }^{(4)}$ devem ser considerados na avaliação da dor no RNPT, tais como o desenvolvimento do sistema nervoso, que configura a localização da dor de acordo com a idade gestacional. O tempo de duração da dor, que pode ser considerada como aguda ou crônica, e efeitos secundários de lesões teciduais, definidas como hiperalgesia e alodínia, demonstrando a complexidade das respostas não verbais emitidas e os fatores que podem estar envolvidos. Entretanto, independente do tipo de dor identificada, a estratégia para o seu alívio deve ser utilizada, podendo ser mediada pela construção de um protocolo institucional para o gerenciamento da dor baseado nas evidências científicas e no cotidiano de cada unidade.

\section{CONCLUSÃO}

A comparação entre o efeito da glicose oral $25 \%$, SNN e as duas intervenções combinadas mostrou que, quando as intervenções são oferecidas de forma combinada, o RNPT é capaz de retornar ao seu estado basal mais rapidamente. Logo, recomenda-se a adoção dessa prática em protocolos assistenciais em unidades neonatais. A importância da avaliação da dor a beira do leito deve ser ressaltada como atividade sistemática por meio de instrumentos capazes de fornecer informações precisas quanto à intensidade da dor e efetividade das intervenções utilizadas para seu alívio à luz do período basal, utilizado como parâmetro de avaliação antes e depois do procedimento doloroso.

\section{RESUMO}

Objetivo: Comparar o efeito da sucção não nutritiva, glicose oral $25 \%$ e glicose oral $25 \%$ combinada com a sucção não nutritiva no alívio da dor de recém-nascidos prematuros submetidos à punção do calcanhar para monitorização da glicemia. Método: Ensaio clínico randomizado crossover, com 34 prematuros que, randomicamente, receberam as intervenções: sucção não nutritiva, glicose oral 25\% e as duas intervenções combinadas durante três dias consecutivos na punção do calcanhar para monitorização da glicemia. Avaliação pelo Premature Infant Pain Profile por 30 segundos antes da intervenção, denominado período basal e por 5 minutos após a punção. Resultados: A combinação das intervenções fez com que os prematuros retornassem ao período basal, com 1 minuto e 30 segundos após a punção do calcanhar, promovendo uma redução percentual de 2,2\% na escala. Conclusão: A comparação do efeito das intervenções isoladas e combinadas mostrou que, quando oferecidas de forma combinada, os prematuros conseguiram retornar aos parâmetros do período basal mais rapidamente. Registro Brasileiro de Ensaios Clínicos: RBR-3gm6w5.

\section{DESCRITORES}

Dor; Recém-Nascido Prematuro; Punções; Comportamento de Sucção; Glucose; Enfermagem Neonatal.

\section{RESUMEN}

Objetivo: Comparar el efecto de la succión no nutritiva, glucosa oral $25 \%$ y glucosa oral $25 \%$ combinada con succión no nutritiva para aliviar el dolor en recién nacidos prematuros sometidos a punción de talón para monitorización de glucosa en sangre. Método: Ensayo clínico cruzado aleatorizado, con 34 prematuros que recibieron aleatoriamente las intervenciones: succión no nutritiva, glucosa oral al 25\% y las dos intervenciones combinadas durante tres días consecutivos en la punción del talón para monitorizar la glucemia. La evaluación por el Premature Infant Pain Profile se realizó durante 30 segundos antes de la intervención, llamado período de línea base 
y durante 5 minutos después de la punción. Resultados: La combinación de intervenciones hizo que los prematuros volvieran a la basal, 1 minuto y 30 segundos después de la punción del talón, promoviendo una reducción porcentual de la escala del 2,2\%. Conclusión: La comparación del efecto de las intervenciones aisladas y combinadas mostró que, cuando se ofrecían en combinación, los recién nacidos prematuros podían volver a los parámetros iniciales más rápidamente. Registro Brasileño de Ensayos Clínicos: RBR-3gm6w5.

\section{DESCRIPTORES}

Dolor; Recien Nacido Prematuro; Punciones; Conducta en la Lactancia; Glucosa; Enfermería Neonatal.

\section{REFERÊNCIAS}

1. Courtois E, Droutman S, Magny J-F, Merchaoui Z, Durrmeyer X, Roussel C et al. Epidemiology and neonatal pain management of heelsticks in intensive care units: EPIPPAIN 2, a prospective observational study. Int J Nurs Stud. 2016;59:79-88. https://doi.org/10.1016/j. ijnurstu.2016.03.014

2. American Academy of Pediatrics. Prevention and management of procedural pain in the neonate: an update. Pediatrics. 2016;137(2):e20154271. https://doi.org/10.1542/peds.2015-4271

3. Witt N, Coynor S, Edwards C, Bradshaw H. A. Guide to pain assessment and management in the neonate. Curr Emerg Hosp Med Rep. 2016;4:1-10. https://doi.org/10.1007/s40138-016-0089-y

4. Kanwaljeet JSA. Defining pain in newborns: need for a uniform taxonomy? Acta Pædiatrica. 2017;106(9):1438-44. https://doi.org/10.1111/ apa.13936.

5. McNair C, Campbell-Yeo M, Johnston C, Taddio A. Nonpharmacologic management of pain during common needle puncture procedures in infants current research evidence and practical considerations: an update. Clin Perinatol. 2019;46(4):709-30. https://doi.org/10.1016/j. clp.2019.08.006

6. Liu Y, Huang X, Luo B, Peng W. Effects of combined oral sucrose and nonnutritive sucking (NNS) on procedural pain of NICU newborns, 2001 to 2016 a PRISMA-compliant systematic review and meta-analysis. Medicine (Baltimore). 2017;96(6):e6108. https://doi.org/10.1097/ MD.0000000000006108

7. Carbajal R, Rousset A, Danan C, Coquery S, Nolent P, Ducrocq S, et al. Epidemiology and treatment of painful procedures in neonates in Intensive Care Units. JAMA. 2008;300(1):60-70. https://doi.org/10.1001/jama.300.1.60

8. Bueno M, Costa P, Oliveira AAS, Cardoso R, Kimura AF. Translation and adaptation of the premature infant pain profile into Brazilian Portuguese. Texto Contexto Enferm. 2013;22(1):29-35. https://doi.org/10.1590/S0104-07072013000100004

9. Axelin A, Salanterä S, Kirjavainem J, Lehtonen L. Oral glucose and parental holding preferable to opioid in pain management in preterm infants. Clin J Pain. 2009;25(2):138-45. https://doi.org/10.1097/AJP.0b013e318181ad81

10. Gradin M, Schollin J. The role of endogenous opioids in mediationg pain reduction by orally administered glucose among newborns. Pediatrics. 2005;115(4):1004-7. https://doi.org/10.1542/peds.2004-1189

11. Gao H, Li M, Gao H, Xu G, Li F, Zhou J et al. Effect of non-nutritive sucking and sucrose alone and in combination for repeated procedural pain in preterm infants: a randomized controlled trial. Int J Nurs Stud. 2018;83:25-33. https://doi.org/10.1016/j.ijnurstu.2018.04.006

12. Carbajal R, Gréteau S, Arnaud C, Guedj R. [Pain in neonatology: non-pharmacological treatment]. Arch Pédiatrie. 2015;22(20):217-21. French. https://doi.org/10.1016/j.arcped.2014.07.001

13. Sposito NPB, Rossato LM, Bueno M, Kimura AF, Costa T, Guedes DMB. Assessment and management of pain in newborns hospitalized in a Neonatal Intensive Care Unit: a cross-sectional study. Rev Latino-Am Enfermagem. 2017;25:e2931. https://doi.org/10.1590/15188345.1665 .2931

14. Gursul D, Hartley C, Slater R. Nociception and the neonatal brain. Semin Fetal Neonatal Med. 2019;24(4):101016. https://doi.org/10.1016/j. siny.2019.05.008 